\title{
Performance of an uncooled imaging interferometric spectrometer with intrinsic background radiation
}

\author{
Ingmar G. E. Renhorn, ${ }^{\mathrm{a}, *}$ Thomas Svensson, ${ }^{\mathrm{b}}$ and Glenn D. Boreman ${ }^{\mathrm{c}}$ \\ ${ }^{a}$ Renhorn IR Consultant AB, Linköping, Sweden \\ ${ }^{b}$ FOI-Swedish Defence Research Agency, Linköping, Sweden \\ ${ }^{c}$ University of North Carolina, Department of Physics and Optical Science, Charlotte, \\ North Carolina, United States
}

\begin{abstract}
A signal-to-noise performance of an imaging Michelson interferometer based on corner cubes is modeled and experimental results are presented. The influence from the background radiation of the uncooled sensor is explained. The noise equivalent spectral radiance is determined in the 700 to $1300 \mathrm{~cm}^{-1}$ range at two spectral resolutions using blackbody sources. The performance of the sensor system is tested on a scene at an average temperature of $19^{\circ} \mathrm{C}$. From these results, the performance is given in terms of number of samples and spectral resolution. The performance model is used to predict the sensor performance at different scene temperatures. (C) The Authors. Published by SPIE under a Creative Commons Attribution 4.0 Unported License. Distribution or reproduction of this work in whole or in part requires full attribution of the original publication, including its DOI. [DOI: 10.1117/1.OE.60.3.033106]
\end{abstract}

Keywords: imaging spectroscopy; corner-cube Michelson interferometer; long-wave infrared; signal-to-noise ratio.

Paper 20210058 received Jan. 14, 2021; accepted for publication Mar. 17, 2021; published online Mar. 30, 2021.

\section{Introduction}

The properties of the corner-cube interferometer were analyzed at an early stage. ${ }^{1}$ An imaging corner-cube Michelson spectrometer has been designed and presented., ${ }^{2,3}$ This design is more compact than the Sagnac configuration, less sensitive to beamsplitter imbalance, and easy to align. However, the corner cubes must have good wavefront quality and proper alignment to maintain high modulation efficiency. It is important that the fore-optics design limits the potential vignetting effects by positioning the entrance pupil of the imaging lens inside the interferometer. ${ }^{4}$ The technology has subsequently been applied in field detection experiments. ${ }^{5}$

The signal-to-noise and imaging characteristics of interferometric instruments compared to dispersive spectrometers are important issues. In remote sensing applications, it is a challenge to find a common figure of merit. ${ }^{6}$ Fellgett and Jacquinot advantages are often cited for interferometric instruments although these advantages come with various caveats. In the thermal spectral region, dispersive instruments are sensitive to internal radiation from the slit mount, grating losses, etc. Interferometric instruments without a slit can be designed to be more flux efficient. For room temperature sensors, internal back-radiation from the sensor into the interferometer and back to the sensor is sometimes a dominating factor. It can be shown that with object temperatures below the intrinsic sensor temperature, interferograms with reversed sign are obtained. At equal temperature, the signal may almost disappear depending on potential difference in emissivity between the intrinsic source and the object being studied. The spectral signal obtained from the measurement must be corrected for the internal source to obtain the spectrum of the object. An interesting class of sensors are the microbolometer cameras. Even for these high temperature sensors, the sensitivity can be $<30 \mathrm{mK}$ at $F / 1$ optics and at a frame rate of $30 \mathrm{~Hz}{ }^{7}$

Establishing the signal-to-noise ratio (SNR) for the uncooled interferometric systems is of considerable interest. Although such systems have been studied over several decades, the SNR related to remote sensing applications is not fully understood. This paper is an effort to support the future application-oriented development of such systems. The importance of background

*Address all correspondence to Ingmar G. E. Renhorn, ingmar.renhorn@telia.com 
radiation is shown and modeled. The effect of the choice of scanning mode (static scene or scanned scene) and the resulting difference in SNR is described. The results should be useful in selecting proper applications for this technology.

In the next section, the theoretical model of the sensor system is presented. Experimental results and model agreements are presented in Sec. 3. Results are discussed and conclusions given in Sec. 4.

\section{Sensor System Model}

The imaging spectrometer presented here can be designed for remote sensing in two different modes. In the staring mode, the interferogram is obtained by moving the corner cube along the $x$ axis as shown in Fig. 1. In the static Fourier transform spectrometer, the interferogram is obtained by scanning the scene over the field of view (FOV). ${ }^{8}$ For a double-sided interferogram, the zero-path-difference (ZPD) fringe is positioned close to the center of the image. Every position in the scene is tracked over the FOV and in this way the interferograms of all scene positions are obtained. The noise characteristics is in this case not only dependent of the temporal noise characteristics of the individual pixels but also on the quality of the nonuniformity correction and the stability over time of this correction. The scene scanning direction is described by the $y$ axis in the models as follows.

Considering an incident plane monochromatic wave with irradiance $E_{0}$, the irradiance at the detector for an optical path difference (OPD) equal to $x$, where $x$ is the OPD from the ZPD point, is given by ${ }^{9}$

$$
E(x)=\frac{\tau_{1}+\tau_{2}}{2} E_{0}\left[1+\frac{2 \sqrt{\tau_{1} \tau_{2}}}{\tau_{1}+\tau_{2}} \cos \left(\frac{2 \pi}{\lambda} x\right)\right],
$$

where $\tau_{1}$ and $\tau_{2}$ are the total transmissions in the two respectively arms. If wavenumbers (where $\tilde{\sigma}=1 / \lambda)$ are used, this equation becomes

$$
E(x)=\tau_{b} E_{0}[1+m \cos (2 \pi \tilde{\sigma} x)],
$$

where $\tau_{b}=\frac{\tau_{1}+\tau_{2}}{2}$ and $m=\frac{2 \sqrt{\tau_{1} \tau_{2}}}{\tau_{1}+\tau_{2}}$ are coefficients corresponding to the overall bias component and the modulation depth, respectively. If $\tau_{1}=\tau_{2}$, the modulation $m$ becomes unity. Ideally

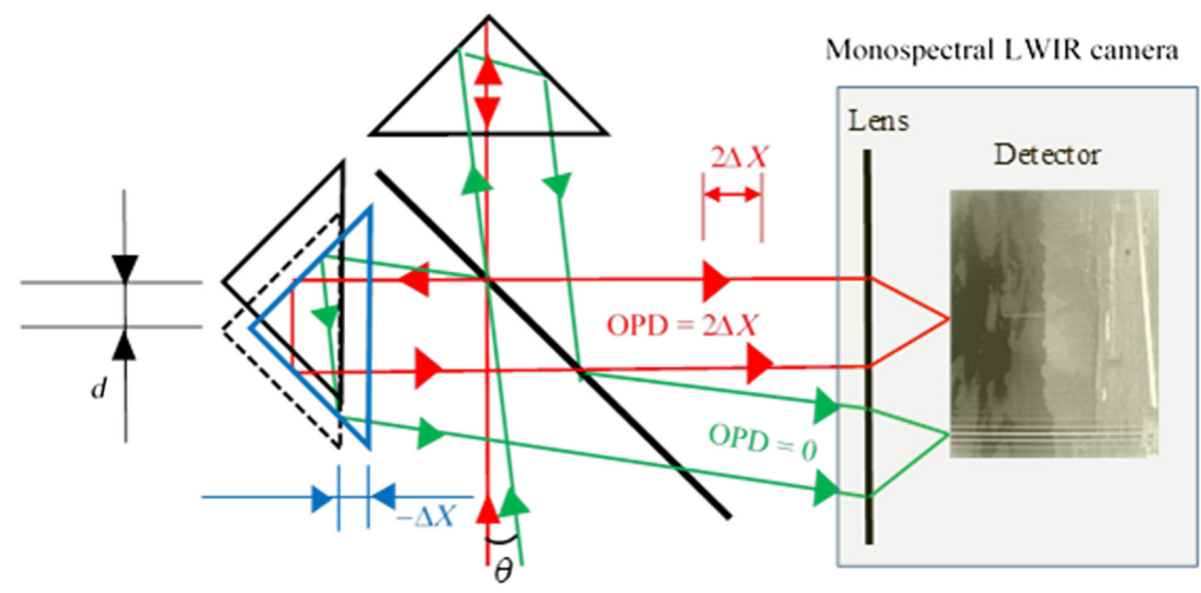

Fig. 1 A schematic sketch of beam paths and scanning of a scene. The two corner cubes marked black (solid lines) show the symmetry position. An asymmetry is introduced by a lateral shift, $d$ (the dashed lines corner cube). A lateral shift $d$ results in OPDs between transmitted and reflected beams for incident angles $\theta \neq 0$ deg [Eq. (4)]. By moving the left corner cube along the optical axis (to the left, $+\Delta X$, or to the right, $-\Delta X$ ), the position of the ZPD fringe will be moved over the array [Eq. (5)]. In the figure, the ZPD fringe has been moved downward by moving the left corner cube to the right (the corner cube marked blue). A scanning of a scene is performed using a stepper motor, enabling a uniform movement of the left corner cube along the optical axis. 
Renhorn, Svensson, and Boreman: Performance of an uncooled imaging interferometric spectrometer...

$\tau_{1}=\tau_{2}=1 / 2$. Phase errors due to corner cube imperfections can lower the modulation depth. ${ }^{10}$ The polarization properties of the corner cubes might also influence the modulation depth. ${ }^{11}$

Allowing for an unknown phase-distortion function, Eq. (2) now becomes

$$
E(x)=\frac{1}{2} E_{0}[1+\cos (2 \pi \tilde{\sigma} x+\varphi)]
$$

where the phase function $\varphi$ could arise from asymmetric sampling and residual dispersion in the optical system. If the incident plane wave is at an angle $\theta$ with respect to the optical axis, the angle-dependent OPD is given as

$$
y=\sin (\theta) 2 d
$$

where $d$ is the corner cube translational distance orthogonal to the optical axis. Including the angular dependence, the equation becomes

$$
E(z)=\frac{1}{2} E_{0}[1+\cos (2 \pi \tilde{\sigma} z+\varphi)]
$$

where $z=x+y$. For each $y$-position, the ZPD can be obtained by adjusting $x$. For a broadband spectrum, the irradiance at the detector is given by the integral

$$
E(z)=\int_{0}^{\infty} \frac{1}{2} E_{0}(\sigma)[1+\cos (2 \pi \tilde{\sigma} z+\varphi)] \mathrm{d} \sigma .
$$

In order to simplify processing using the complex Fourier transform, a symmetric function with both negative and positive wavenumbers is introduced: ${ }^{12}$

$$
E(z)=\int_{-\infty}^{\infty} E_{0}(|\tilde{\sigma}|) \cos (2 \pi \tilde{\sigma} z+\varphi) \mathrm{d} \sigma,
$$

where the mean value has been subtracted. The corresponding inverse Fourier transform of the interferogram $E(z)$ is

$$
E_{0}(\tilde{\sigma}) \exp (i \varphi)=\int_{-\infty}^{\infty} E(z) \exp (i 2 \pi \tilde{\sigma} z) \mathrm{d} z
$$

where now $E_{0}(\tilde{\sigma})=\left|E_{0}(\tilde{\sigma}) \exp (i \varphi)\right|$.

Intrinsic radiation originating from the detector array must be considered. This contribution can, for an uncooled sensor, be larger than the object radiation. If this radiation is $F_{0}(\tilde{\sigma})$, the interferogram is ${ }^{13}$

$$
E(z)=\int_{-\infty}^{\infty}\left[E_{0}(|\tilde{\sigma}|)-F_{0}(|\tilde{\sigma}|)\right] \cos (2 \pi \tilde{\sigma} z+\varphi) \mathrm{d} \tilde{\sigma}
$$

If $F_{0}>E_{0}$, the interferometric signal will be negative at ZPD. The corresponding spectrum is obtained from

$$
E_{0}(|\tilde{\sigma}|)-F_{0}(|\tilde{\sigma}|)=\left|\int_{-\infty}^{\infty} E(z) \exp (i 2 \pi \tilde{\sigma} z) \mathrm{d} z\right|
$$

These two sources can be separated by calibrating against a low temperature blackbody and a high temperature blackbody. The spectrometer signal is given as

$$
\begin{array}{ll}
S_{1}(\tilde{\sigma})=R(\tilde{\sigma})\left[F_{0}(\tilde{\sigma})-E_{0}(\tilde{\sigma})\right] & \text { if } F_{0}(\tilde{\sigma})>E_{0}(\tilde{\sigma}), \\
S_{2}(\tilde{\sigma})=R(\tilde{\sigma})\left[E_{0}(\tilde{\sigma})-F_{0}(\tilde{\sigma})\right] & \text { if } E_{0}(\tilde{\sigma})>F_{0}(\tilde{\sigma}),
\end{array}
$$

where $R(\tilde{\sigma})$ is the spectral responsivity of the instrument at specified number of samples and bandwidth. 
Assuming a low temperature blackbody target in the first measurement, i.e., $S_{1}$, and a high temperature blackbody in the second measurement, i.e. $S_{2}$, the following relation is obtained

$$
\begin{gathered}
R(\tilde{\sigma})=\frac{S_{1}(\tilde{\sigma})+S_{2}(\tilde{\sigma})}{L_{2}(\tilde{\sigma})-L_{1}(\tilde{\sigma})} \\
F_{0}(\tilde{\sigma})=\frac{S_{1}(\tilde{\sigma}) L_{2}(\tilde{\sigma})+S_{2}(\tilde{\sigma}) L_{1}(\tilde{\sigma})}{S_{1}(\tilde{\sigma})+S_{2}(\tilde{\sigma})}
\end{gathered}
$$

where $L_{1}$ and $L_{2}$ are the blackbody radiances at temperatures $T_{1}$ and $T_{2}$, respectively, where $T_{1}<T_{\text {sensor }}$ and $T_{2}>T_{\text {sensor }}$. The blackbody radiance is given as

$$
L(\tilde{\sigma})=\frac{2 h c^{2} \tilde{\sigma}^{3}}{\exp \left(\frac{h c \tilde{\sigma}}{k T}\right)-1},
$$

where $h$ is the Planck constant, $c$ is the speed of light, and $k$ is the Boltzmann constant. The wavenumber $\tilde{\sigma}$ is here given in SI units. The scale must be changed to obtain the unit of $\mathrm{cm}^{-1}$ common among spectroscopists.

The interferogram is obtained by sampling over the OPD from $-l$ to $l$ relative to the ZPD at equidistant positions. If the stepping size is $\delta x$ and $N$ samples are obtained on each side of the $\mathrm{ZPD}$, the total number of samples is $2 N+1$. The maximum OPD is $l=N \delta x$. The maximum wavenumber, $\tilde{\sigma}_{\max }$, is obtained from $\tilde{\sigma}_{\max }=1 / \delta x$. The Nyquist wavenumber is half this value or $\tilde{\sigma}_{\text {Nyquist }}=1 /(2 \delta x)$. The wavenumber step is $\delta \tilde{\sigma}=\tilde{\sigma}_{\max } /(2 N)=1 /(2 l)$. This is also the common definition of spectral resolution. In practice, there are many ways that this resolution might be compromised, primarily from deficiencies in the optics.

The Fourier transform of the sampled interferogram is given as

$$
E_{s}(\tilde{\sigma})=\left|\frac{1}{\sqrt{2 N+1}} \sum_{r=1}^{2 N+1} E_{r}(z) \exp \left[\frac{i 2 \pi(r-1)(s-1)}{2 N+1}\right]\right|,
$$

where the zero-wavenumber term appears at position 1 . This transformation preserves the signal power. This means, e.g., that for a random signal, the transformed signal preserves the same power. This is useful when discussing SNRs. Taking the absolute value of a complex number however changes the statistical properties. If the initial random noise is a normal probability distribution with variance $\sigma_{n}^{2}$, the transformed noise will have a Rayleigh distribution. The Rayleigh probability density function is given by

$$
\operatorname{PDF}_{\text {Rayleigh }}(s)=s \exp \left(-\frac{s^{2}}{2 \sigma_{R}^{2}}\right)
$$

where the variance is given by $\sigma_{R}^{2}=(2-\pi / 2) \sigma_{n}^{2}$ and $\sigma_{n}^{2}$ is the initial sensor-signal noise variance. The spectral signal bias of the Rayleigh distribution is bias $=\sqrt{\pi / 2} \sigma_{n}$. These results can be used in calculating the expected spectral SNR at given number of samples and spectral resolution.

The spectral SNR (denoted with subscript of $\tilde{\sigma}$ ) at a specific object radiance is given by

$$
\operatorname{SNR}_{\tilde{\sigma}}=\frac{S_{0}(\tilde{\sigma})}{\sigma_{R}}=\frac{L_{0}(\tilde{\sigma})}{N E S R_{\tilde{\sigma}}}
$$

and

$$
\operatorname{NESR}_{\tilde{\sigma}}=\frac{L_{0}(\tilde{\sigma})}{S N R_{\tilde{\sigma}}}=\frac{\sigma_{R}}{R(\tilde{\sigma})},
$$

where NESR is the noise equivalent spectral radiance required in the object plane and $S_{0}(\tilde{\sigma})=R(\tilde{\sigma}) L_{0}(\tilde{\sigma})$. The noise equivalent temperature difference (NETD) can be obtained from 


$$
\operatorname{NETD}_{\tilde{\sigma}}=\frac{\operatorname{NESR}_{\tilde{\sigma}}}{d L(\tilde{\sigma}) / d T},
$$

where NETD is commonly determined at a blackbody temperature of $20^{\circ} \mathrm{C}$.

The optimal number of channels depends on the spectral structure of the target. If the only difference between samples is a wavenumber-independent emissivity, only separation in terms of a graybody emissivity is needed. This is still a quite challenging task since atmospheric absorption and emission and reflected radiation have to be taken into account. Often the object surface is assumed to be diffuse making reflected radiation independent of surface orientation. This obviously does not apply to specular surfaces, for which the cloud cover variation can be of importance. Often, however, the downwelling radiation is neglected. It is also assumed that the range variation in the scene is small. It is important to include spectral regions with low atmospheric absorption, i.e., bands close to $10 \mu \mathrm{m}$. To obtain emissivity for each channel, a supplementary assumption has to be added. Often the observation that many solid objects show a smooth emissivity behavior is used to make the problem solvable.

If scenes are obtained at short ranges, the atmospheric absorption can be ignored. With the limited SNR that can be obtained with an uncooled system, the practical application is on separating objects in the scene due to their combined temperature and emissivity variation.

The sensor framerate is often set to a fixed value, usually the maximum framerate allowed by the thermal time constant. An important factor is the total time for obtaining a spectral image. With a fixed recording time and fixed framerate, the number of frames is determined. Given a fixed number of frames, how is the system optimized for a particular purpose? This will depend on the spectral range and the required Nyquist wavenumber. Aliasing is avoided by the filtering effect of the detector spectral responsivity. This also means that only the spectral region where the sensor spectral responsivity is high (resulting in reasonable SNR) is of interest. The minimum wavenumber limit $\tilde{\sigma}_{\max }=2 \tilde{\sigma}_{\text {Nyquist }}$ where the Nyquist wavenumber is at the onset of aliasing effects. With a spectral resolution of $\delta \tilde{\sigma}$, the number of one-sided samples are $N=\tilde{\sigma}_{\max } /(2 \delta \tilde{\sigma})$. Table 1-3 give experimental settings for the sensor system as presented here.

With a framerate of $25 \mathrm{~Hz}$, the following recording times as given in Table 2 are obtained.

The SNR varies for the four cases, as expected. The normalized SNR varies as given in Table 3 .

Table 1 Number of samples at maximum wavenumber.

\begin{tabular}{lcc}
\hline \hline Bandwidth $\left(\mathrm{cm}^{-1}\right)$ & No. of samples $\tilde{\sigma}_{\max }=4000\left(\mathrm{~cm}^{-1}\right)$ & No. of samples $\tilde{\sigma}_{\max }=12,000\left(\mathrm{~cm}^{-1}\right)$ \\
\hline 25 & $2 \times 80+1$ & $2 \times 240+1$ \\
50 & $2 \times 40+1$ & $2 \times 120+1$ \\
\hline \hline
\end{tabular}

Table 2 Recording times corresponding to the conditions given in Table 1.

\begin{tabular}{lcc}
\hline \hline Bandwidth $\left(\mathrm{cm}^{-1}\right)$ & Recording time $\tilde{\sigma}_{\max }=4000$ & Recording time $\tilde{\sigma}_{\max }=12,000$ \\
\hline 25 & $6.44 \mathrm{~s}$ & $19.24 \mathrm{~s}$ \\
50 & $3.24 \mathrm{~s}$ & $9.64 \mathrm{~s}$ \\
\hline \hline
\end{tabular}

Table 3 SNR variation normalized to the highest value.

\begin{tabular}{lcc}
\hline \hline Bandwidth $\left(\mathrm{cm}^{-1}\right)$ & Relative SNR $\tilde{\sigma}_{\max }=4000$ & Relative SNR $\tilde{\sigma}_{\max }=12,000$ \\
\hline 25 & 0.41 & 0.71 \\
50 & 0.57 & 1.00 \\
\hline \hline
\end{tabular}


From these settings, it seems beneficial to not set the Nyquist wavenumber any higher than necessary. This could conflict with the bandwidth requirements with respect to resolving spectral features to obtain adequate spectral discrimination. The SNR result can therefore not be taken as the only criterion for optimizing the acquisition of the scene's spectral information. This will be discussed further below.

\section{Experimental Methods}

The interferometric imaging sensor using an uncooled detector array is shown in Fig. 2. The spectrum can be obtained by either scanning one of the corner cubes along its axis or by scanning the image along a panoramic scene. In the latter case, the responsivity of each pixel must be the same to a high accuracy. This is difficult to achieve by a nonuniformity correction (NUC) process, and therefore some SNR degradation is expected. The noise properties are slightly different for the two cases but should be qualitatively similar. Most of the analysis below is done using the scanning of one of the corner cubes.

The setup is a slightly modified standard Michelson configuration. As described in Ref. 2, the two plane mirrors were substituted with two front-surface (Au coated) corner-cube reflectors, of 63.5-mm diameter, purchased from Edmund Scientific. Specified retroreflection parallelism was 1 second of arc. The beamsplitter and a nominally identical compensator plate, purchased from II-VI Infrared, were made from $\mathrm{ZnSe}$, in rectangular format $70 \mathrm{~mm} \times 100 \mathrm{~mm} \times 7 \mathrm{~mm}$. One surface of the beamsplitter was a nominal 50/50 LWIR beamsplitter coating, while the other surface (and those of the compensator) was coated with a broadband antireflector, with $<1 \%$ reflection across the 8 to $14 \mu \mathrm{m}$ band. The two $\mathrm{ZnSe}$ plates were mounted together, separated by metallic shims of about $0.25-\mathrm{mm}$ thickness, with the beamsplitter coating on an interior surface.

The uncooled LWIR camera, A655SC (FLIR Systems), has a microbolometer focal-plane array. It has $640 \times 480$ pixels, a pixel pitch of $17 \mu \mathrm{m}$ and a spectral response between 7 and $14 \mu \mathrm{m}$. The NETD is $<30 \mathrm{mK}$ and the digital output range is 14 bits. The focal length of the $F / 1.0$ lens is $41.3 \mathrm{~mm}$, providing an FOV of $\sim 11 \mathrm{deg} \times 15 \mathrm{deg}$. The maximum full frame rate is $50 \mathrm{~Hz}$.

The frame rate during scanning was typically set to 25 or $50 \mathrm{~Hz}$. The translation speed of the left corner cube along the optical axis during scanning was $0.01 \mathrm{~mm} / \mathrm{s}$, provided by the stepper motor, TDC001 (Thorlabs). A schematic sketch of the scanning mechanism of the interferometric imaging sensor was shown in Fig. 1.

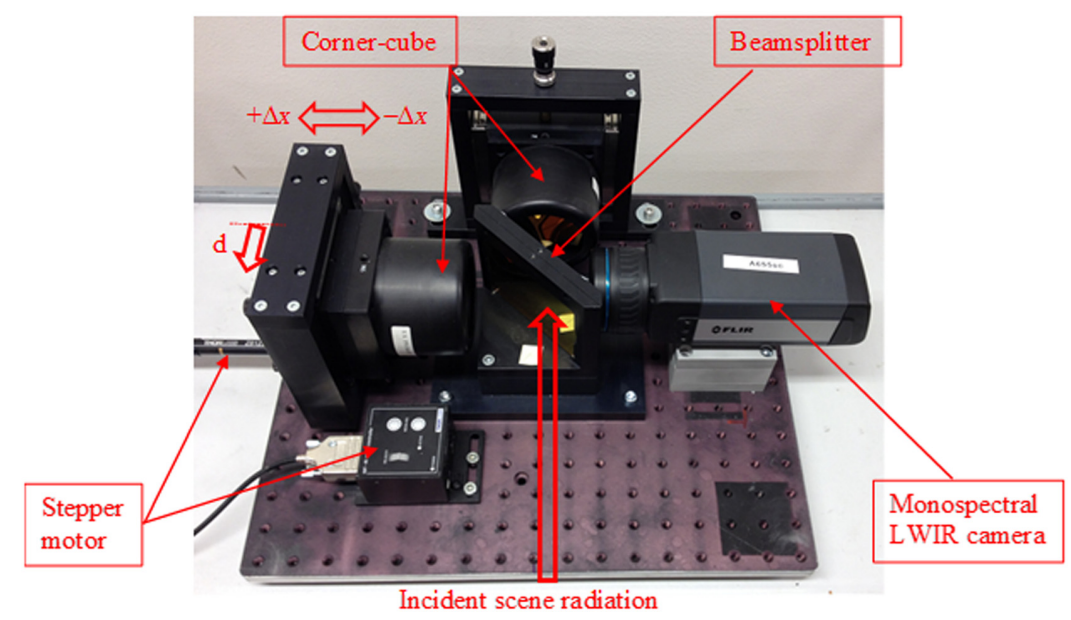

Fig. 2 The hyperspectral camera (with a protective cover removed). The basic parts are two corner-cube mirrors: a beamsplitter and an LWIR uncooled camera. A lateral shift $d$ is introduced in the left corner-cube mirror, which can be moved along its (optical) axis by a stepper motor (Thorlabs TDC001) where $\pm \Delta x$ denotes differences from the ZPD position. 
There are two ways of obtaining an interferogram. When one of the corner cubes is scanned along its optical axis, a temporal interferogram is obtained at each imaging pixel. Noise is dominated by the temporal noise and not affected by sensor nonuniformities since the spectrum is obtained at each individual pixel. The (Gaussian-distributed) noise of the temporal interferogram is measured to be $\sigma_{x}=4.3 \mathrm{DN}$ where $x$ denotes scanning the corner cube along the $x$-coordinate. The temporal NETD is $30 \mathrm{mK}$ corresponding to $3.4 \mathrm{DN}$. Keeping the corner cubes at fixed positions, the interferogram is obtained by scanning the scene and monitoring the signal from the same object position during this scan. With this approach, there are several errors that can degrade the SNR. The most important one is the ability to obtain NUC of the sensor. Even for a well-behaved system, the noise is expected to increase substantially. In the example shown here in Fig. 3, the noise level is increasing to $\sigma_{y}=10.0 \mathrm{DN}$, where $y$ denotes scanning the scene in the $y$-coordinate direction. The noise is more than doubled. The radiometric correction of the sensor is of outmost importance and in particular in scanning of the scene, which have practical advantages. The spectral signal standard deviation based on Rayleigh statistics is $\sigma_{x, R}=2.8 \mathrm{DN}$ and $\sigma_{y, R}=6.6 \mathrm{DN}$, respectively. There is also a small bias, bias $_{x, R}=5.4 \mathrm{DN}$ and bias $_{y, R}=12.5 \mathrm{DN}$. The test of the experimental system will hereafter be based on scanning the corner cube.

A blackbody source model SR-800R from CI Systems was used for radiance calibration. The signal was first studied for a blackbody target at $60^{\circ} \mathrm{C}$ at a constant nominal spectral resolution of $\delta \tilde{\sigma}=27 \mathrm{~cm}^{-1}$ and varying the number of samples $N_{\text {samples }}=2 N+1$, where $N$ was equal to $N=\{80,120,240\}$. The Nyquist wavenumber will under these conditions be proportional to $N$ and $\tilde{\sigma}_{\text {Nyquist }}=\{2155,3232.5,6465\} \mathrm{cm}^{-1}$. With the Fourier transform as defined above, the signal is proportional to $\sqrt{2 N+1}$. The SNR can be increased by increasing the number of samples. With a constant framerate, this will require increased recording time. With a framerate of $25 \mathrm{~Hz}$, the signals shown in Fig. 4 are obtained after $t_{\text {rec }}=\{3.2,4.8,9.6\} \mathrm{s}$ at optimal sampling.

A higher SNR can be obtained by sampling at a lower spectral resolution. This comes at a cost of poorer ability to resolve narrower bands. Whether this potential loss of information is acceptable depends on the specific application, i.e., the spectral properties of the object under study.

The spectral resolution was set to $\delta \tilde{\sigma}=54 \mathrm{~cm}^{-1}$ and the number of samples set to $N_{\text {samples }}=2 N+1$, where $N$ was equal to $N=\{40,60,120\}$. The Nyquist wavenumbers are

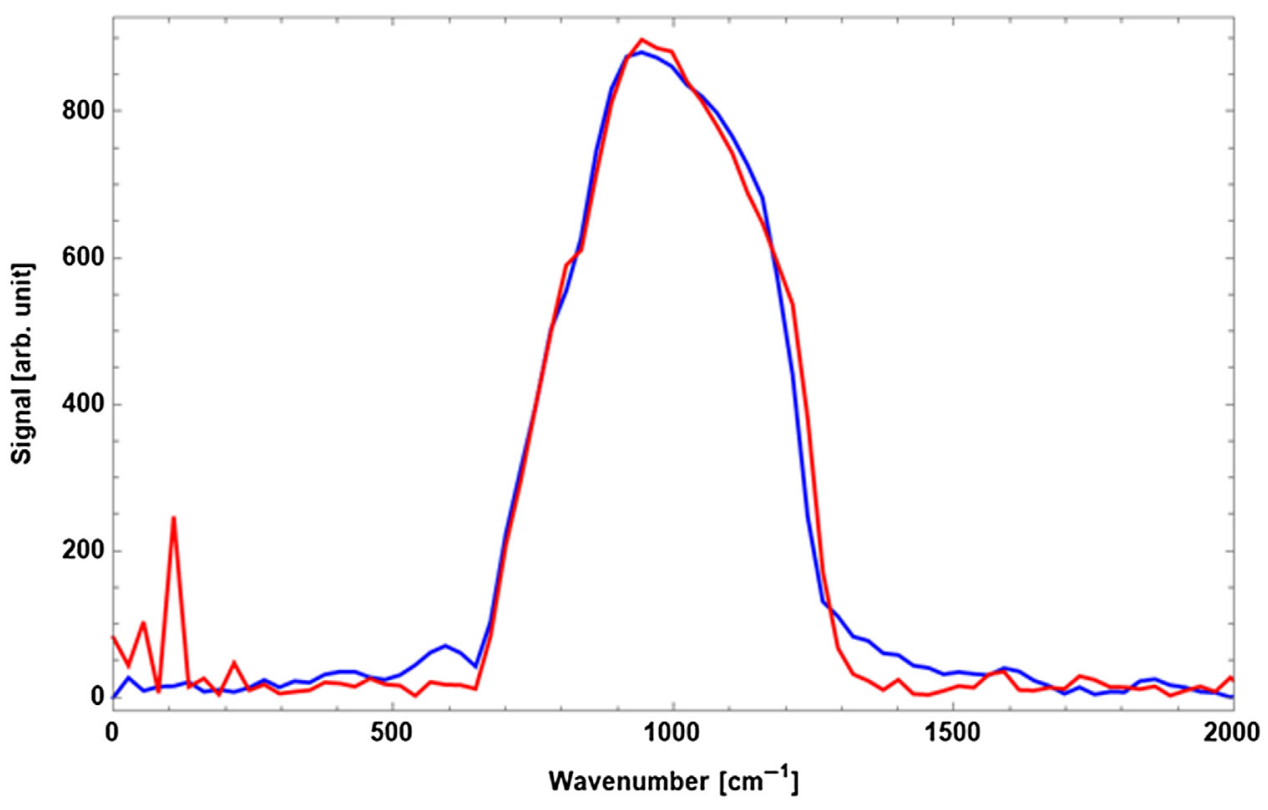

Fig. 3 The same spectrum obtained in two different ways when imaging a blackbody at $60^{\circ} \mathrm{C}$. The blue curve is obtained when translating the corner-cube along the optical axis, i.e., changing the $x$-coordinate while keeping the $y$-coordinate at zero in Eq. (5). The red curve is obtained when scanning in the image plane, i.e., changing the $y$-coordinate while keeping the $x$-coordinate at zero. 


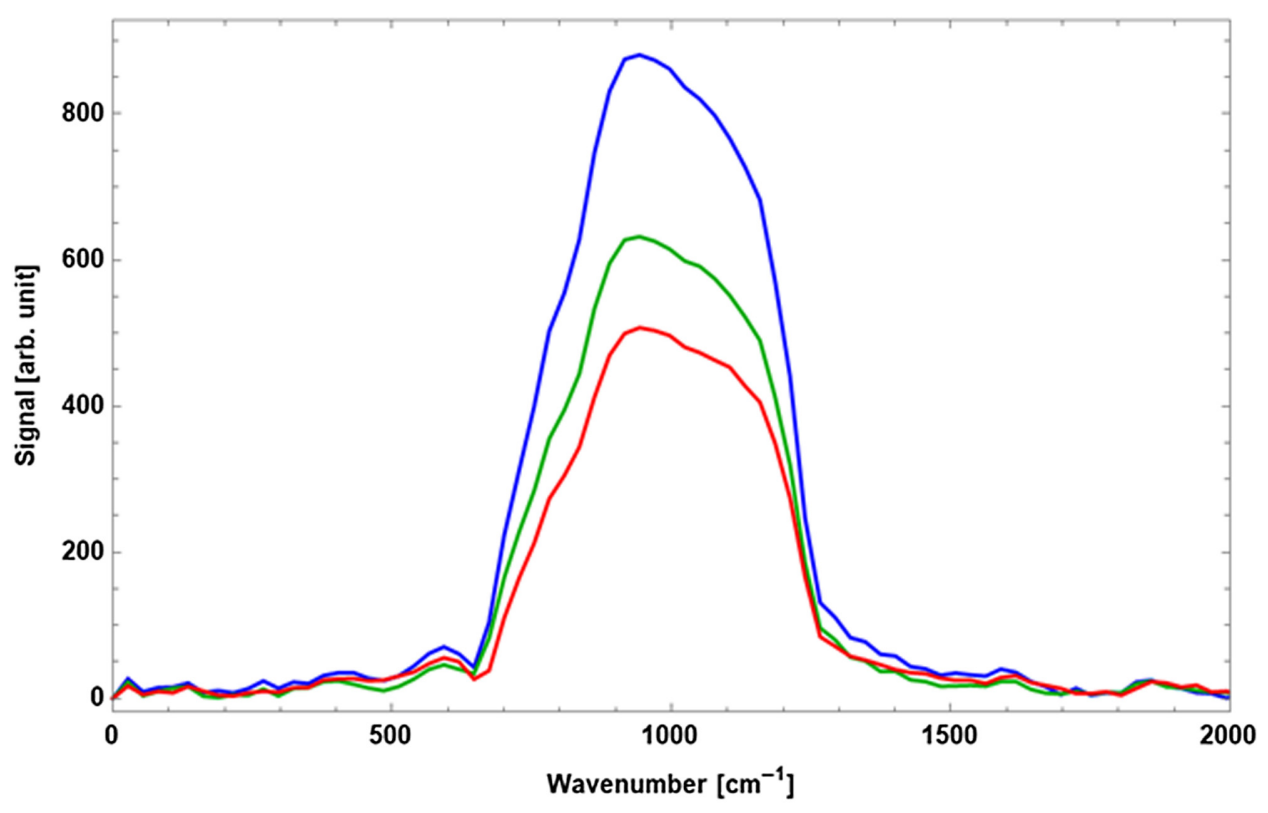

Fig. 4 Instrument signal with a blackbody temperature of $60^{\circ} \mathrm{C}$. Bandwidth is $27 \mathrm{~cm}^{-1}$. Number of one-sided steps, $N$, is $N=80,120$, and 240 for the red, green, and blue curves, respectively. The noise is scaled to stay constant. The signal is proportional to $\sqrt{2 N+1}$. The Nyquist wavenumber is $2155,3232.5$, and $6465 \mathrm{~cm}^{-1}$, respectively. The signal is constituted by two counteracting contributions: the external blackbody radiation and the intrinsic radiation emanating from the sensor.

the same as already mentioned, i.e., $\tilde{\sigma}_{\text {Nyquist }}=\{2155,3232.5,6465\} \mathrm{cm}^{-1}$. Again, the signal is proportional to $\sqrt{2 N+1}$. With a framerate of $25 \mathrm{~Hz}$, the signals shown in Fig. 5 are obtained after $t_{\text {rec }}=\{1.6,2.4,4.8\} \mathrm{s}$ at optimal sampling.

Similar data to those obtained for the $60^{\circ} \mathrm{C}$ blackbody target were also obtained for a $10^{\circ} \mathrm{C}$ target. The sensor temperature is in between these two temperatures, and the sensor radiance can be determined as described already. The resulting sensor spectral radiance is shown in Fig. 6. These data were compared to blackbody radiation, and the corresponding sensor temperature was determined to $32^{\circ} \mathrm{C}$. This radiation is back injected into the interferometric system and will always be present since the sensor is stabilized at this temperature. This must be considered when deriving the final spectral signal from any object of interest.

Simultaneously when determining the sensor radiation, the spectral response of the sensor can be obtained by Eq. (12). The result is shown in Fig. 7. The relative responsivity is in good agreement with published typical response for this type of sensors.

Correcting the signal with respect to the back-injected radiance from the sensor, the target spectrum can be obtained. This is illustrated for the target at $10^{\circ} \mathrm{C}$ in Fig. 8 . The agreement is reasonable considering the type of sensor involved.

The correction due to the back-radiation from the uncooled sensor is important to obtain reliable radiometric measurements. There is a concern that radiation emanating from the inside of the sensor system could result in interferences with a phase variation of $S_{1}$ that deviates from $S_{2}$ in Eq. (12). A common procedure in practice is to use the absolute value of each parameter. If both $S_{1}$ and $S_{2}$ have the same phase dependence, this works well. Phase error due to asymmetric sampling relative to the ZPD is here disregarded. However, there is a possibility that radiation emanating from the internal parts of the interferometer, i.e., $S_{2}$, can result in phase variations that deviate from those experienced by $S_{1} \cdot{ }^{14}$ This is especially the case when the emitter is placed between the sensor and the interferometer. ${ }^{15}$ The interferogram resulting from the radiation from the sensor is expected to have a phase difference of $\pi$ radians relative to the radiation from the target. If this is exactly true, the simple procedure using absolute values as described above still works. If there are deviations from this ideal case, the complex-valued processing in Ref. 14 could improve results. It is therefore of interest to quantify the magnitude of possible errors for each interferometer design. The responsivity was calculated using Eq. (10) in Ref. 14. 
Renhorn, Svensson, and Boreman: Performance of an uncooled imaging interferometric spectrometer...

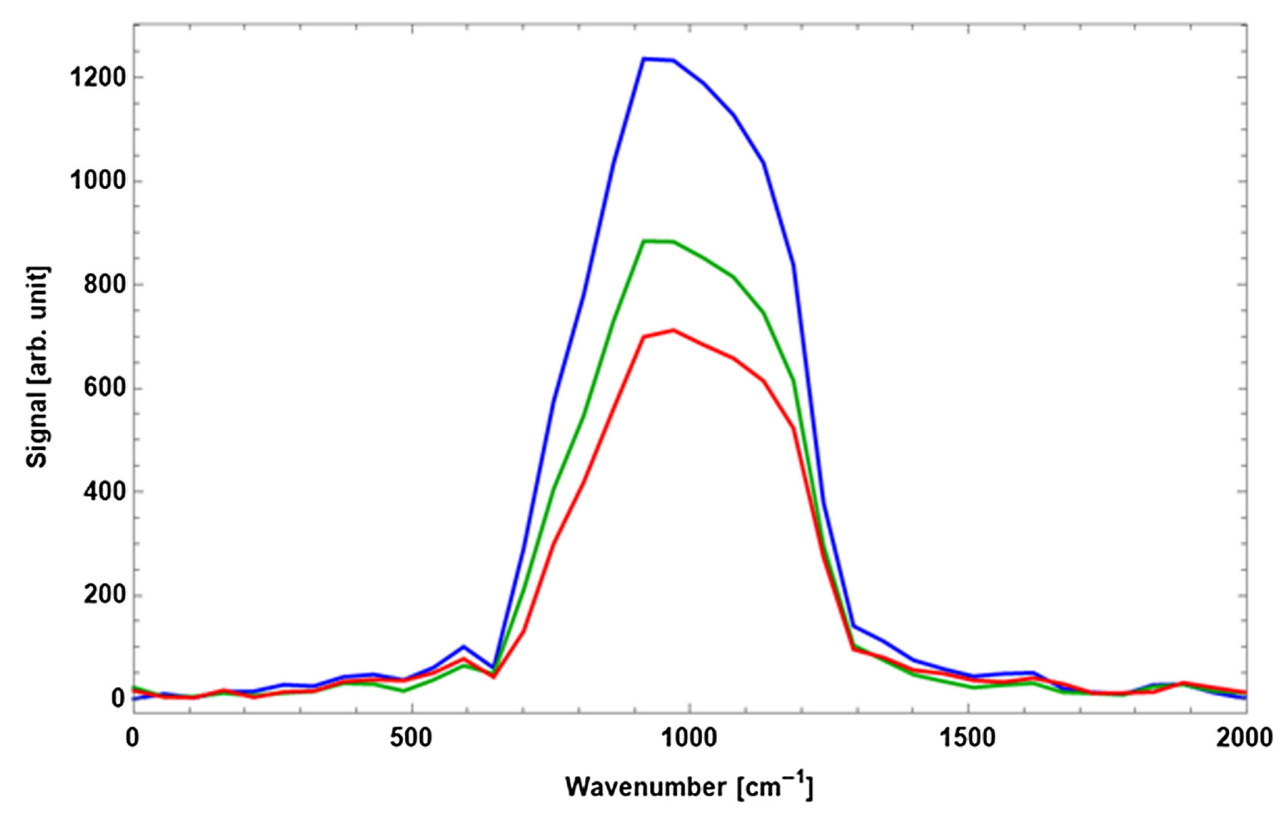

Fig. 5 Corresponding curves to Fig. 4 but with a bandwidth of $54 \mathrm{~cm}^{-1}$. Number of one-sided steps, $N$, is $N=40,60$, and 120 for the red, green, and blue curves, respectively. The Nyquist wavenumber is $2155,3232.5$, and $6465 \mathrm{~cm}^{-1}$, respectively. The signal is proportional to $\sqrt{2 N+1}$. The SNR is increasing with larger bandwidth compared to Fig. 4 at the expense of spectral resolution. If this is a gain or loss depends on the spectral properties of the object under study.

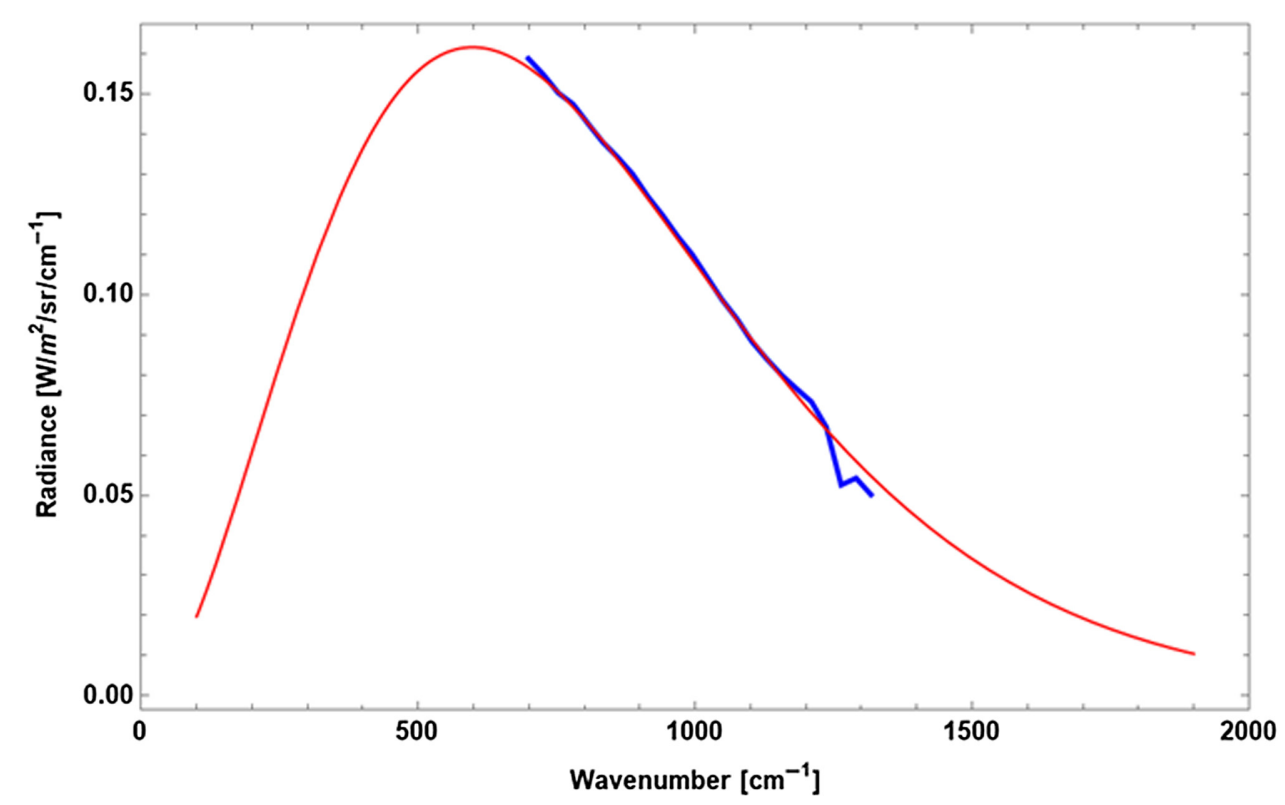

Fig. 6 The sensor radiance is here shown in the blue curve. By combining signals from the $10^{\circ} \mathrm{C}$ and the $60^{\circ} \mathrm{C}$ blackbodies, the internal sensor radiance can be determined as described above. The sensor temperature is determined to $32^{\circ} \mathrm{C}$ and represented by the red curve. Since the sensor is temperature stabilized at this temperature, this internal radiation will always be present in the measurements. 


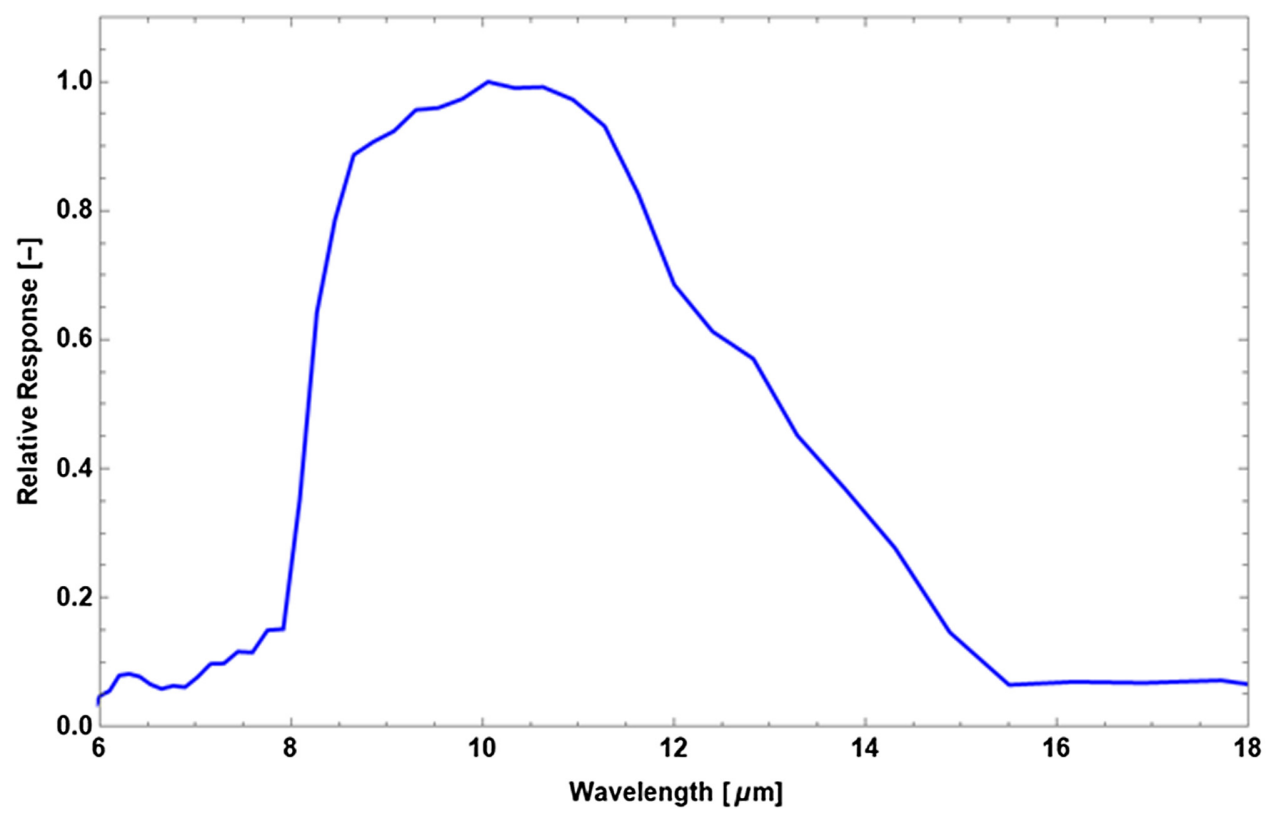

Fig. 7 The detector responsivity can be determined in the same way as the internal radiation. The relative response is shown as a function of wavelength to make comparison with published sensor data easier.

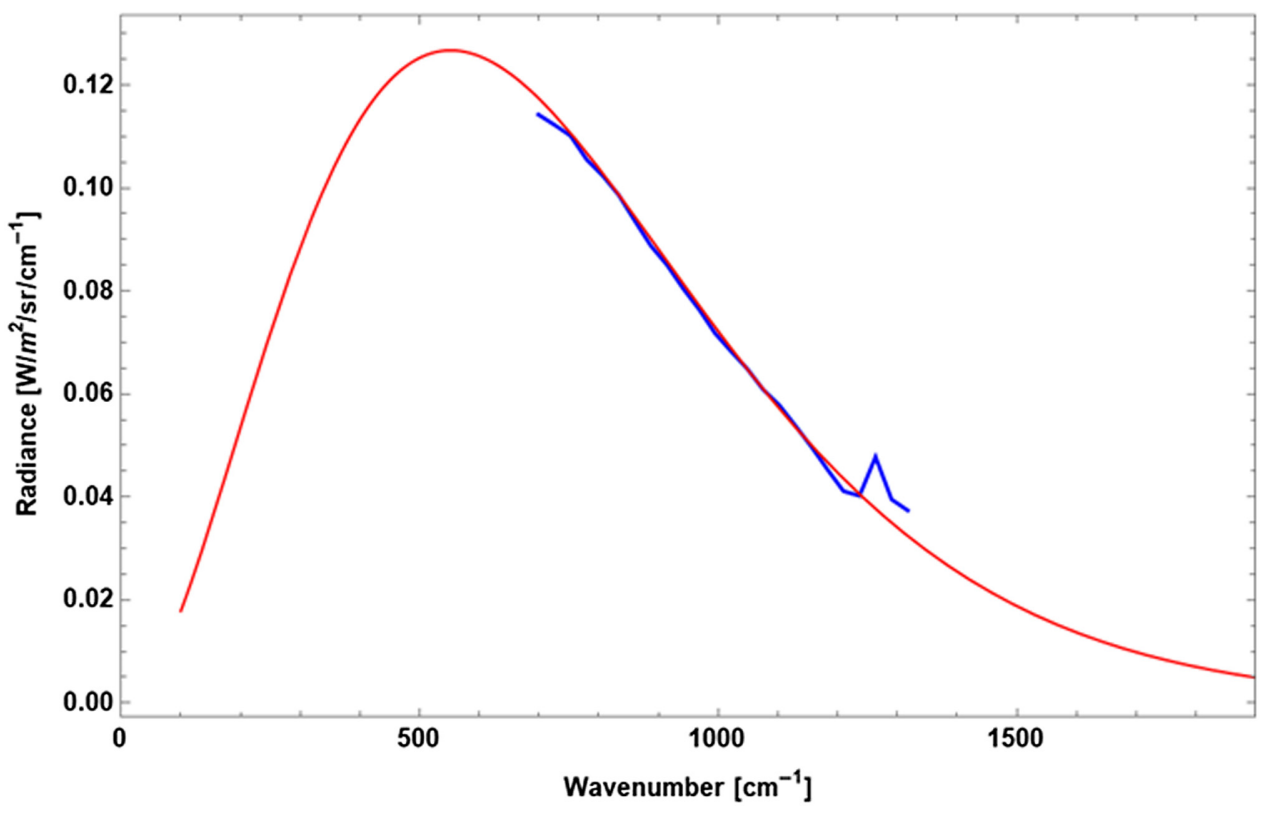

Fig. 8 Taking the internal radiation into account, the corresponding object radiance can be calculated. The measured radiance from the blackbody with the nominal temperature $10^{\circ} \mathrm{C}$ is shown. The theoretical radiance curve is shown in red for the temperature $8.3^{\circ} \mathrm{C}$.

The difference between the result above using absolute values and the complex-valued treatment is $<0.5$ per cent. Also, the phase variation in $S_{2}$ was measured using Eq. (7) of Ref. 14. The phase varied between -0.15 and $0.07 \mathrm{rad}$. Also differences in spectral results are negligible.

The noise statistics will be slightly different. The variance is almost equal for both cases while the bias obtained when using absolute values is absent when using the complex-valued method.

The temporal NESR measurement shown in Fig. 9 was obtained at two different measurements with spectral resolutions, $27 \mathrm{~cm}^{-1}$ at 240 samples and $54 \mathrm{~cm}^{-1}$ at 120 samples and 


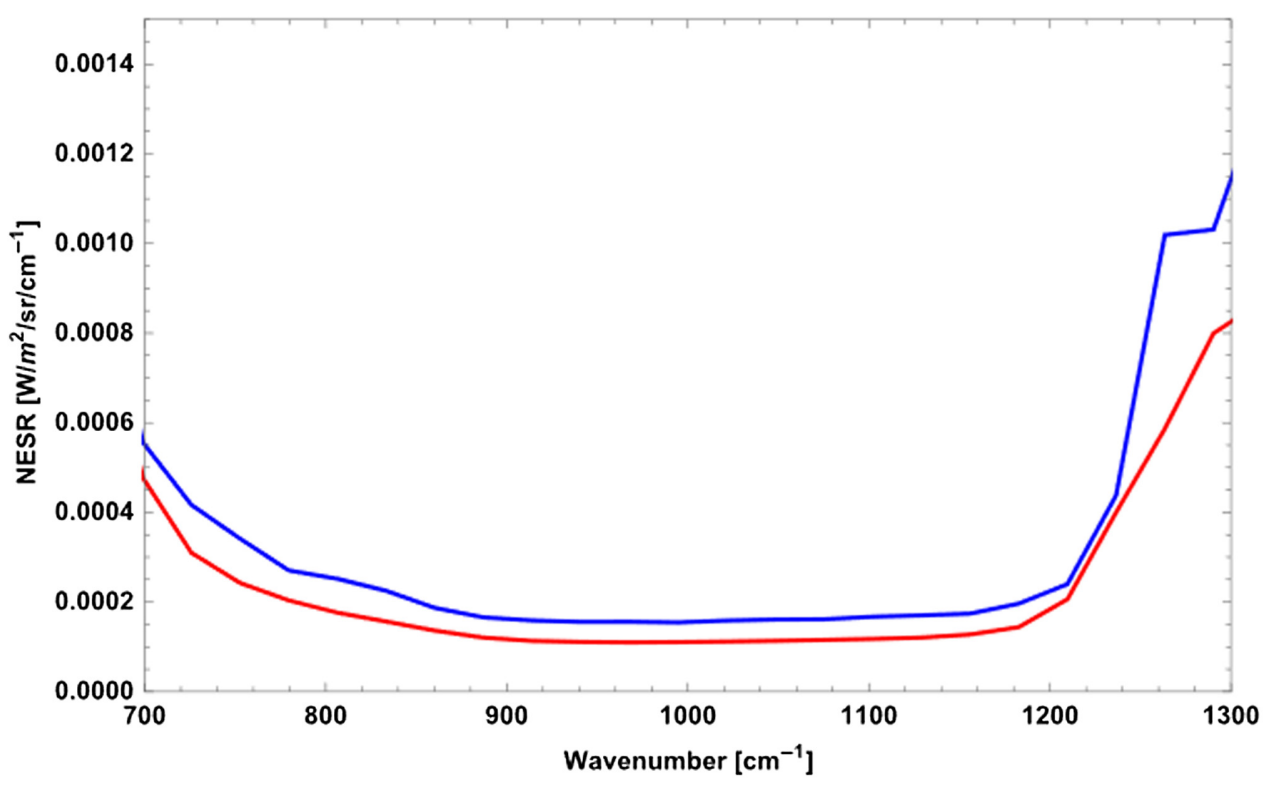

Fig. 9 NESR obtained from responsivity. The bandwidth is $27 \mathrm{~cm}^{-1}$ for the blue curve and the number of one-sided steps is 240 . The bandwidth is $54 \mathrm{~cm}^{-1}$ for the red curve and the number of one-sided steps is 120 .

measured sensor noise properties. The result scales well with the assumption that responsivity can be modelled as $R(\tilde{\sigma})=R_{0}(\tilde{\sigma}) \sqrt{2 N+1} \delta \tilde{\sigma}$. Under these conditions, the responsivity at the larger bandwidth is a factor $\sqrt{2}$ larger than the smaller bandwidth. This does, however, not mean that the larger bandwidth contains more information. The advantage in using a larger spectral bandwidth applies only for targets with spectral features broad enough to be resolved. The expected spectral features of the object must be considered when selecting appropriate spectral resolution to achieve an optimal number of uncorrelated spectral bands.

According to Eq. (17), the SNR can be obtained from the NESR results with respect to the temperature of the scene. An example is given in Fig. 10 for a scene under consideration. This example is for the case of scanning the corner cube.

Since the sensor is far from BLIP, it is assumed that the back-injected internal radiation does not contribute to the observed noise in any considerable way.

These kinds of sensors are most frequently used as scene scanning instrument where the spatial part of the interferogram is interrogated. As seen in Fig. 11, the noise level is then increased by more than a factor of 2 due to limited capability in NUC. This limitation is difficult to compensate for by oversampling since the nonuniformities require frequent renewing of the NUC calibration that interrupts the recording of the scene data. In any event, accurate NUC procedures are needed to obtain satisfactory results.

Although the larger bandwidth has a higher SNR that does not necessarily mean that it contains more information. This depends on the spectral variation in the scene. An indication of these conditions is given in the example below. The scene temperature is estimated to $19^{\circ} \mathrm{C}$. Figure 12 is obtained at a bandwidth of $54 \mathrm{~cm}^{-1}$ and Fig. 13 is obtained at a bandwidth of $27 \mathrm{~cm}^{-1}$. It can be observed that the performance is remarkably similar in the two cases although the SNR is higher in Fig. 12 compared to Fig. 13. The three-color components are obtained by selecting specific components from a PCA. The components are for both Figs. 12 and 13 as follows: blue channel:PC1; green channel: PC3; and red channel: PC6. The results depend on the type of signal processing selected; a more detailed analysis of those issues is beyond the scope of this paper.

An average scene spectral signal is adopted and all objects in the scene are treated as a deviation from this spectral signal. This simplifies the detection algorithm when only relative changes are being considered. In Fig. 14, a comparison is shown between the average scene spectral signal and a set of spectral values of the target shown in yellow in Figs. 12 and 13. 
Renhorn, Svensson, and Boreman: Performance of an uncooled imaging interferometric spectrometer...

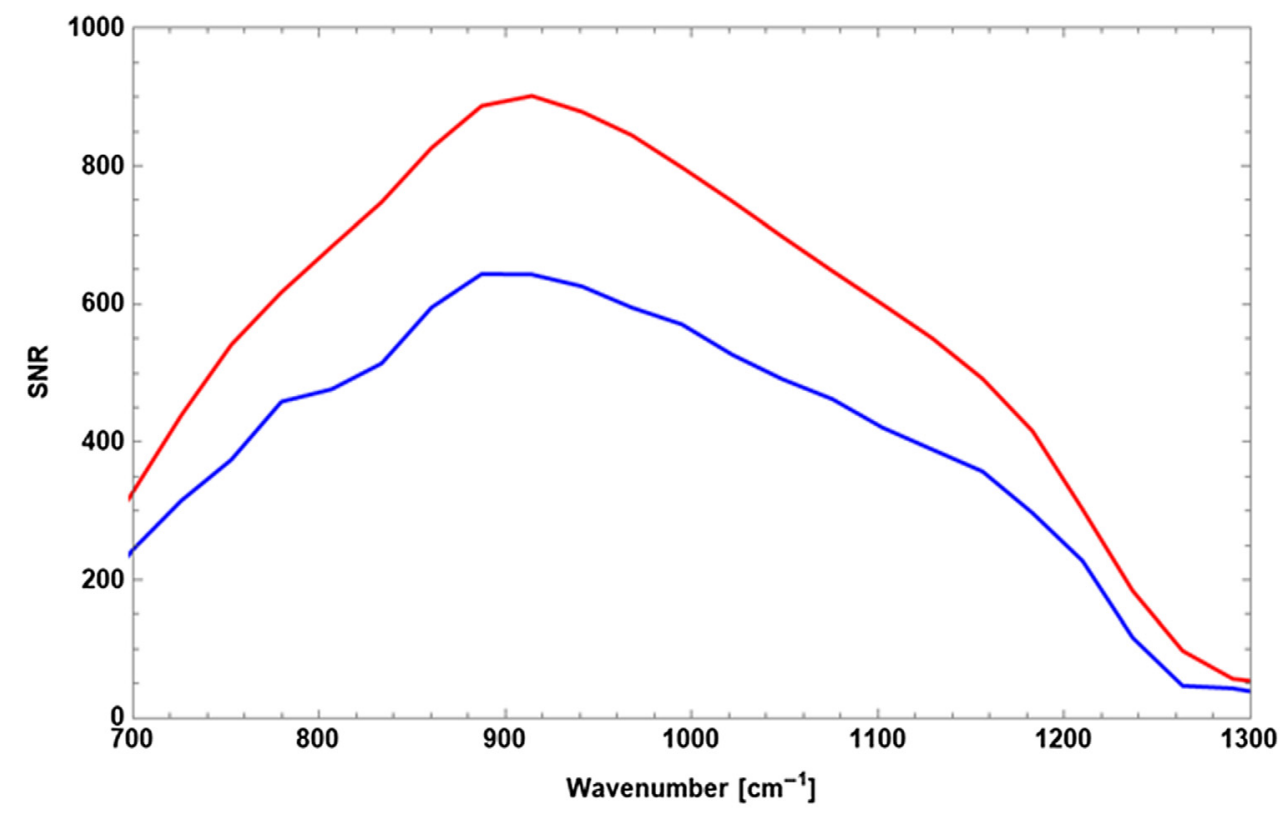

Fig. 10 SNR for an object at $19^{\circ} \mathrm{C}$ at the bandwidth $27 \mathrm{~cm}^{-1}$ and $N=240$ (blue curve) and $54 \mathrm{~cm}^{-1}$ and $N=120$ (red curve).

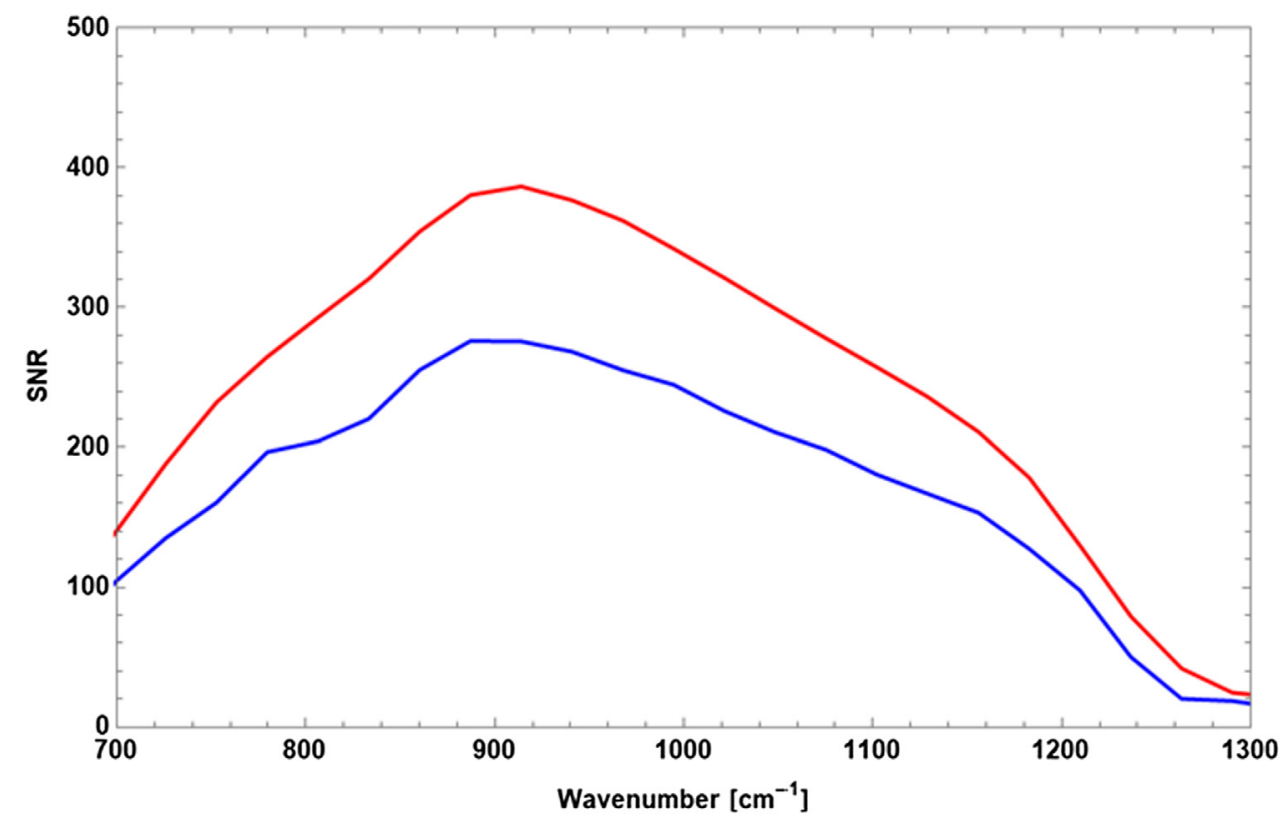

Fig. 11 Same conditions as in Fig. 10 but noise level at scene scanning is shown for comparison.

The broader spectral band signal (green in Fig. 14) has been scaled by a $\sqrt{2}$ to obtain the same signal levels as for the narrower spectral band (red in Fig. 14). As can be observed, there are some spectral variations that distinguish the target from average scene signal although the noise level is substantial at this SNR. The SNR obtained in these measurements are therefore considered to be a minimum for useful results.

The scene in Fig. 15 was obtained in the summer at sunset with the targets in shadow. The distance to the targets is about $20 \mathrm{~m}$ and the camera is placed $12 \mathrm{~m}$ above ground. The small round disc to the left consists of crumpled aluminum foil (high reflectivity material), the red plate is made of granite (silicate rich material), and the green plate is made of limestone (carbonate rich 
Renhorn, Svensson, and Boreman: Performance of an uncooled imaging interferometric spectrometer...

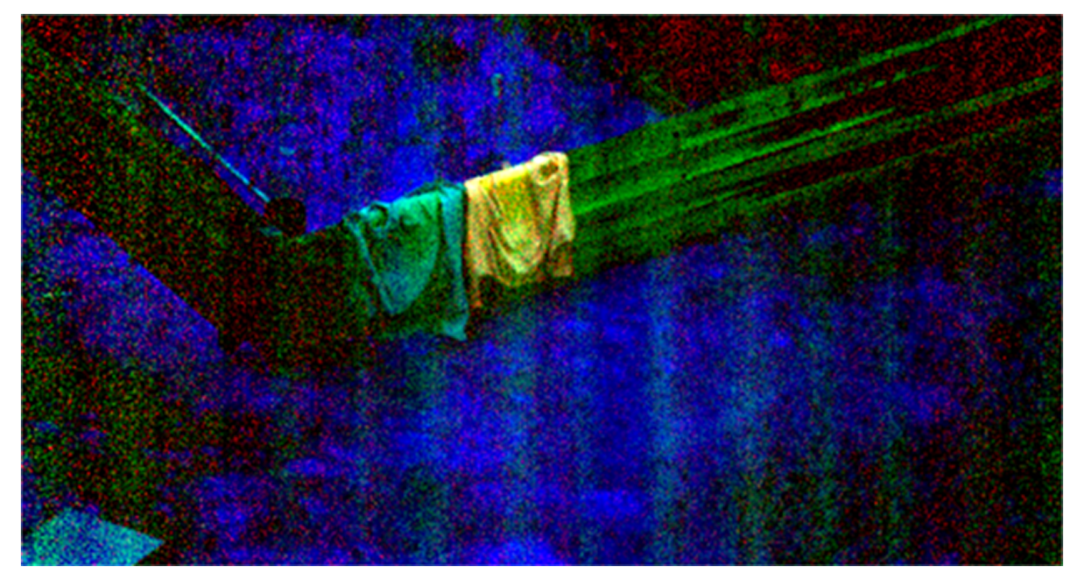

Fig. 12 Selected PCA-components are shown of a scene recorded at $54 \mathrm{~cm}^{-1}$ and $N=120$.

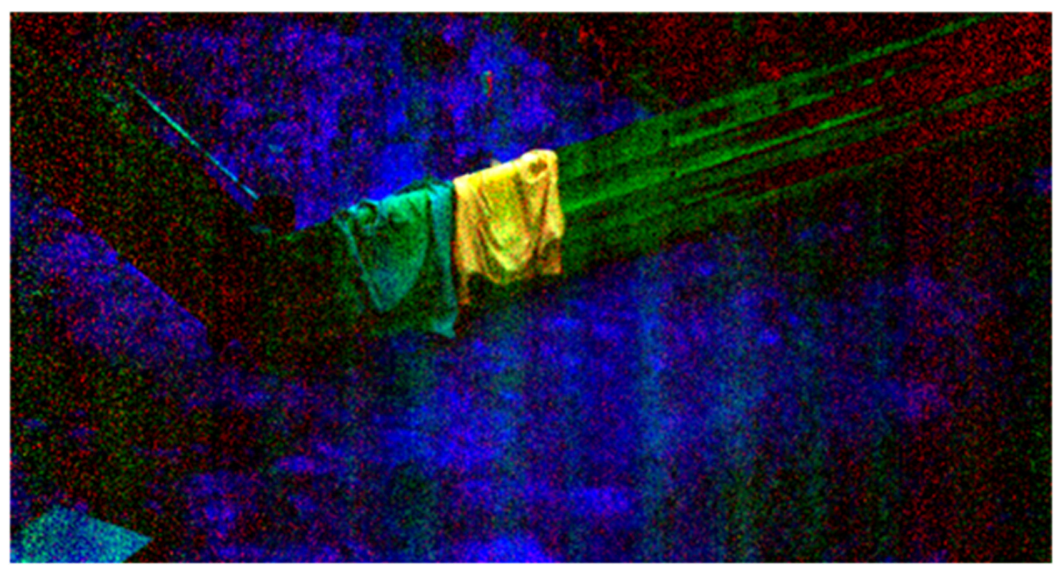

Fig. 13 The same selected PCA-components as above are shown of the same scene recorded at $27 \mathrm{~cm}^{-1}$ and $N=240$.

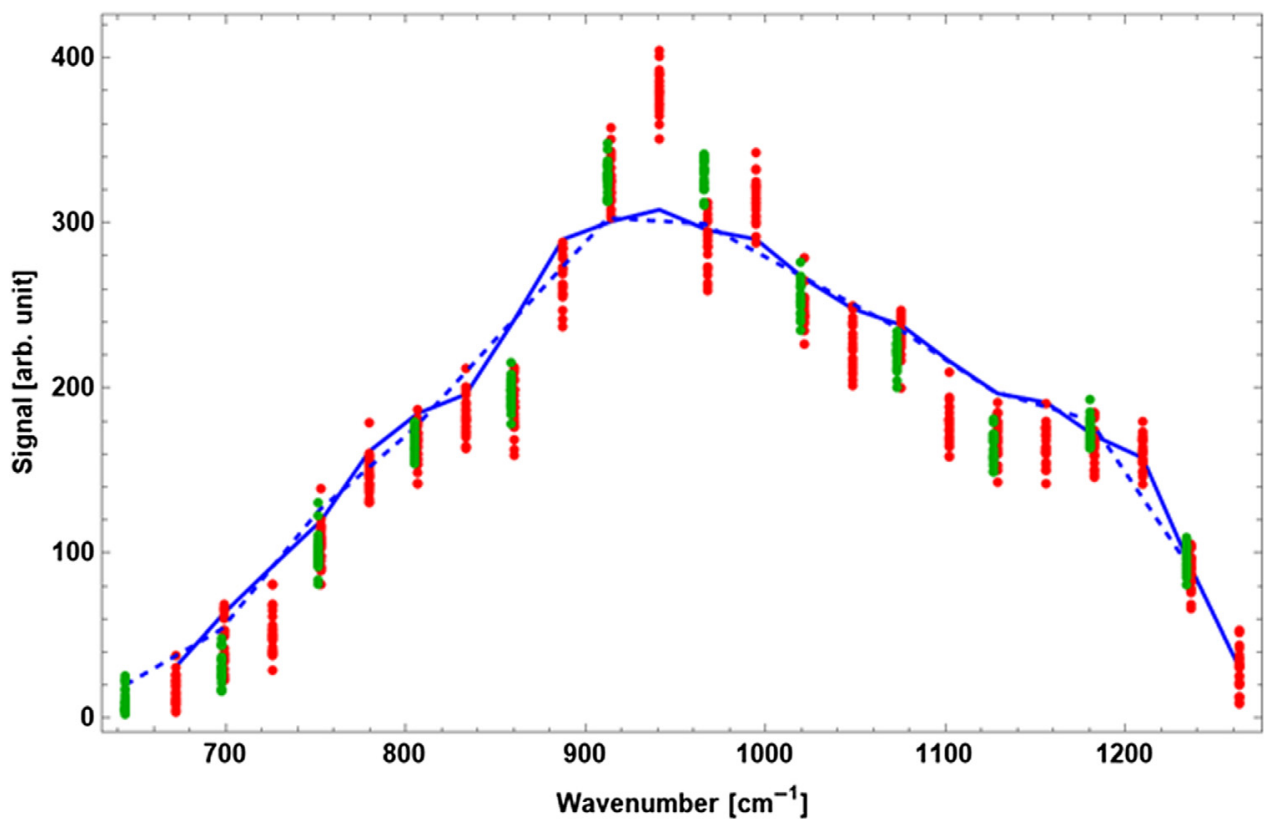

Fig. 14 Blue curve is average scene spectral signal (dashed for the broader spectral bands). Green datapoints (scaled by a factor $\sqrt{2}$ ) are signal distribution of the yellow target in Fig. 12 and red datapoints are signal distribution of the same target in Fig. 13. 


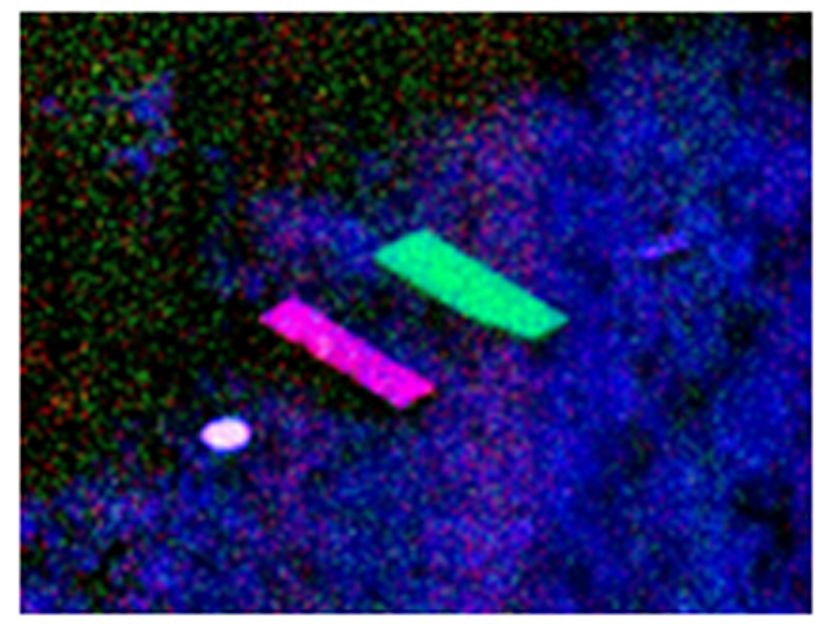

Fig. 15 Objects on a grass field.

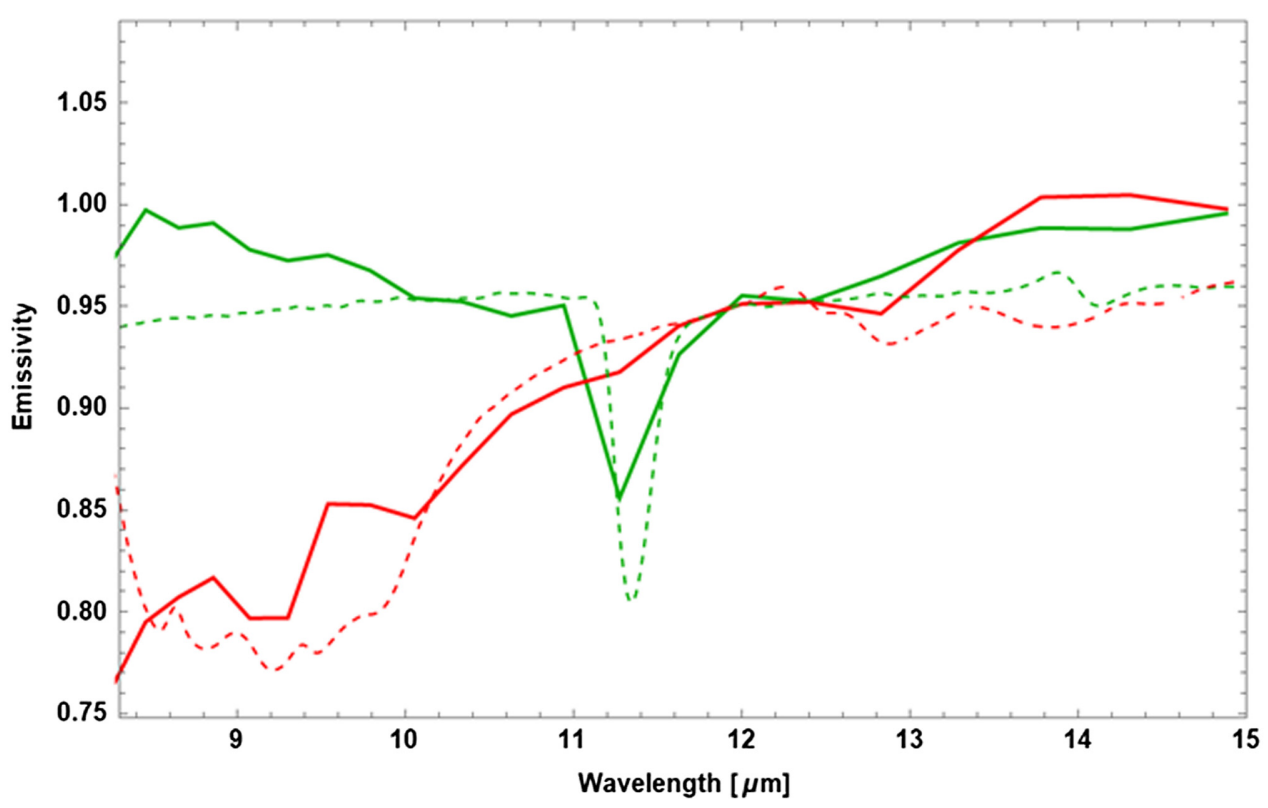

Fig. 16 Emissivity distribution of the two targets of color indicated in Fig. 15. Dashed curves are from laboratory reflectance measurements.

material). The blue background is a grass field with low reflectivity. As in the previous example, an average spectral signal of the scene is calculated and all objects in the scene are treated as a deviation from this spectral signal. The principal components shown in Fig. 15 are: blue channel: PC1; green channel: PC7; and red channel: PC5.

In Fig. 16, the emissivity is derived by comparing the target radiance with a blackbody radiance at the estimated scene temperature $20^{\circ} \mathrm{C}$. The green targets show a dip in emissivity at $11.2 \mu \mathrm{m}$, typical for limestone while the red target shows an increase in emissivity with wavelength, typical for silicate-rich material.

\section{Discussion and Conclusion}

A theoretical model and experimental results are reported for the imaging spectrometer described. The interferometric system design was presented in Ref. 2 and is here modified using an uncooled imaging sensor with low NETD. The camera uses a standard lens, which is why vignetting is not avoided but is compensated for using blackbody sources in flat-field correction. 
Renhorn, Svensson, and Boreman: Performance of an uncooled imaging interferometric spectrometer...

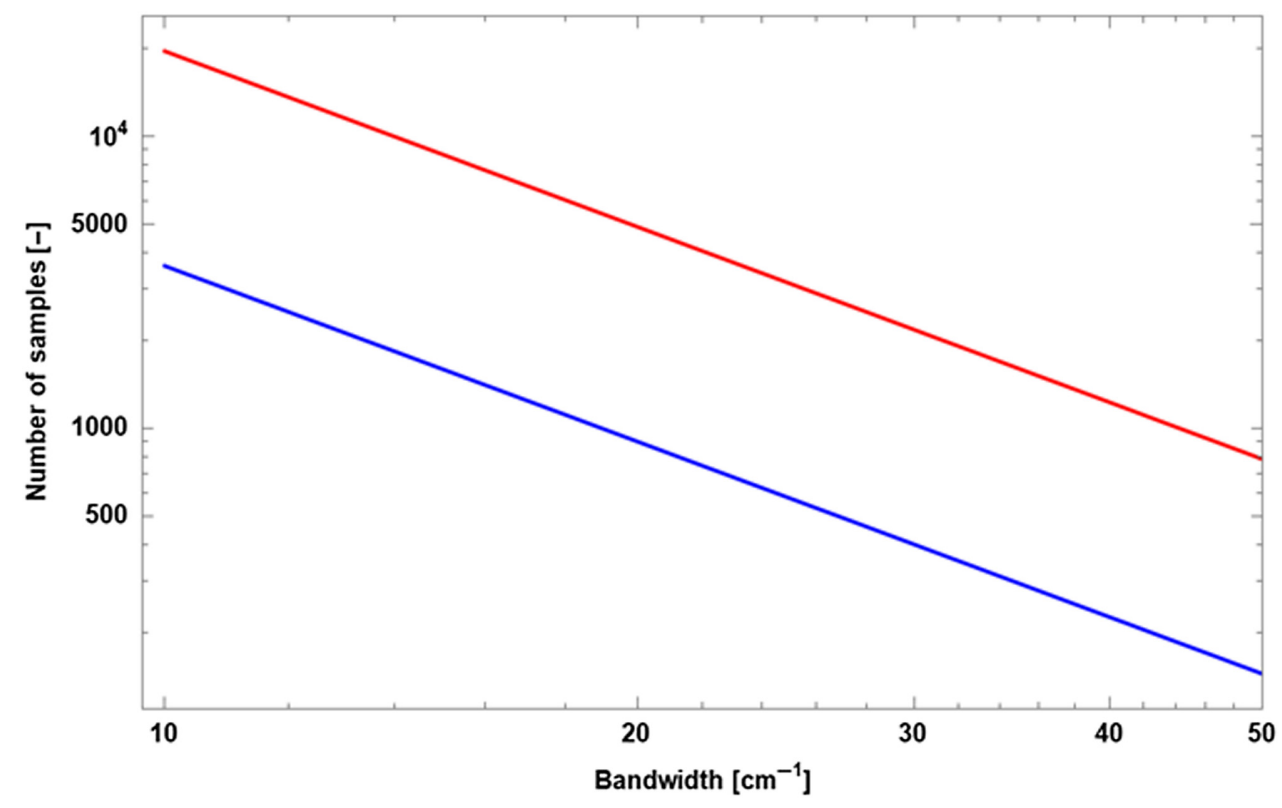

Fig. 17 Boundary for the number of samples at a given bandwidth as defined above. Scene temperature is $19^{\circ} \mathrm{C}$. The blue curve is for scanning the corner cube and the red curve is for a scene scan. The importance of accurate nonuniformity corrections is obvious.

Vignetting can be avoided using lenses with the pupil positioned at the entrance of the corner cubes. ${ }^{16}$

The effect of NUC instability is different for the data obtained by scanning the scene compared to data obtained by scanning one of the corner cubes. Scanning the corner cube results in a higher SNR, provided the scene and the sensor platform are static. If the sensor is placed on an airborne vehicle, limitations in pointing accuracy will likely degrade the performance. For a scene scanning system, internal radiometric calibration and frequent NUC might be required.

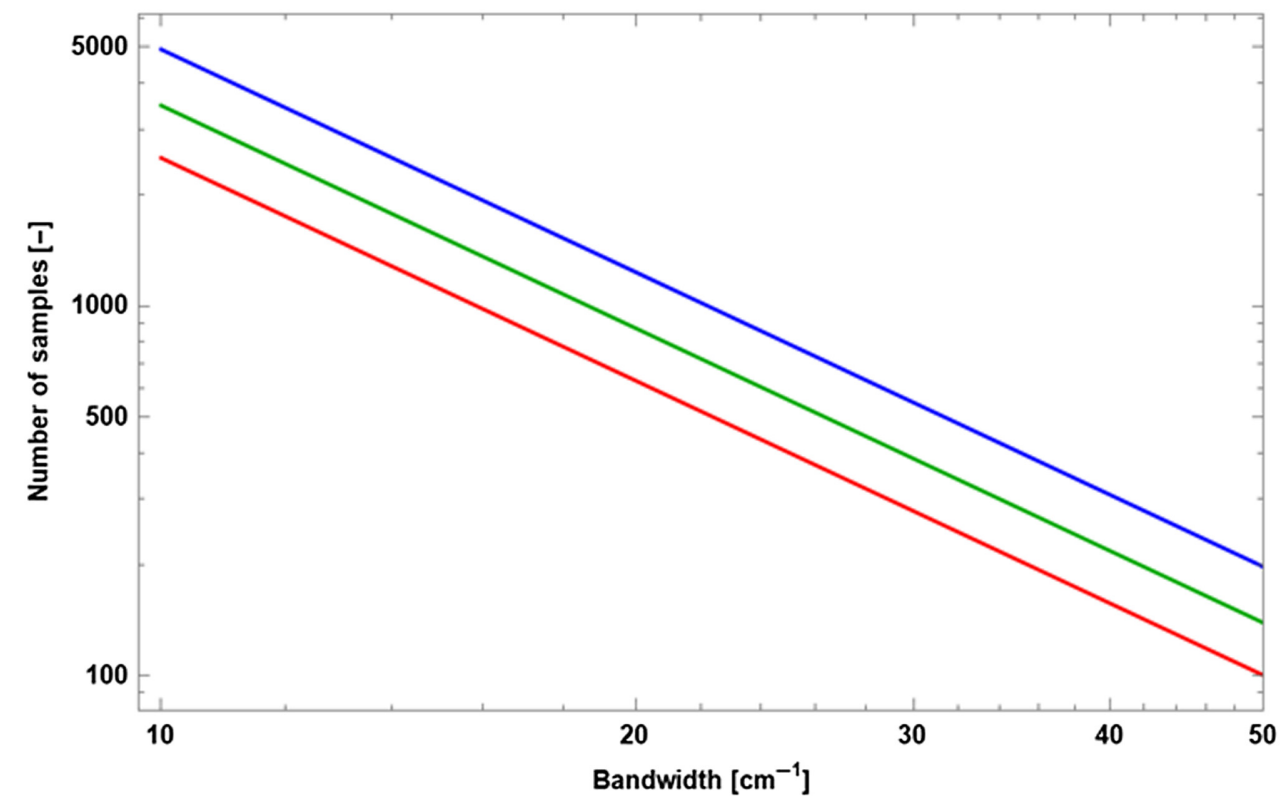

Fig. 18 Number of samples as a function of bandwidth at different scene temperatures. Blue curve is at $10^{\circ} \mathrm{C}$, green curve at $20^{\circ} \mathrm{C}$, and red curve at $30^{\circ} \mathrm{C}$. The curves are representative for the corner cube scanning system. 
Besides FPA nonuniformities, limitations can be introduced by registration errors from processing the interferometric image sequence in a scene scanning recording. Registration can be supported by supplementary measurements with an inertial measurement unit (IMU), external cameras monitoring the scene or the interferometric images themselves. The impact of registration errors depends on the local variation in spectral radiance.

The required spectral resolution depends on the application. For sensing of pollutant clouds, the spectral resolution must be higher than demonstrated in the present experiments ${ }^{17}$ except for gases with broad band signatures such as Freon.

The signal noise is assumed to be dominated by the sensor noise. Besides the sensor noise, the SNR will be determined by the bandwidth and the number of samples. The bandwidth is governed by the scanning range. When scanning the corner cube, the present sensor performance is shown in the blue curve of Fig. 17 where $\sqrt{2 N+1} \delta \tilde{\sigma}=600$. For scene scanning, the corresponding limit is $\sqrt{2 N+1} \delta \tilde{\sigma}=1400$. This applies to a scene at $19^{\circ} \mathrm{C}$. The number of samples is equal to $2 N+1$. In Fig. 17, it can be observed that for high spectral resolution, many samples are required. When separating different types of materials with rather narrow spectral features, the scene must be static and stable over a longer time to allow for these sampling requirements. The relation to the scene temperature is shown in Fig. 18.

From these results, it can be concluded that the technology considered here is useful for static scenes at reasonably high temperatures when a modest spectral resolution is sufficient. The frame rate of the sensor is often fixed, so the number of samples translates into a recording time. This will often be the practical limitation of an application under consideration.

\section{References}

1. E. R. Peck, "Theory of the Corner-Cube interferometer," J. Opt. Soc. A 38(12), 1015-1024 (1948).

2. I. Renhorn et al., "Demonstration of a corner-cube-interferometer LWIR hyperspectral imager," J. Infrared Millimeter Terahertz Waves 31, 60-65 (2010).

3. D. Bergström et al., "Noise properties of a corner-cube Michelson interferometer LWIR hyperspectral imager," Proc. SPIE 7660, 76602F (2010).

4. H. Wang et al., "Optical design of a static LWIR Fourier-transform imaging spectrometer with high throughput," Proc. SPIE 8910, 89100Q (2013).

5. N. Wang et al., "Performance of a long-wave infrared Fourier transform imaging spectrometer using a corner-cube Michelson interferometer and an uncooled microbolometer array," Proc. SPIE 9674, 96740C (2015).

6. T. Skauli, "Specifying radiometric performance of hyperspectral and conventional cameras: a minimal set of independent characteristics," Proc. SPIE 11392, 1133920B (2020).

7. A. Rogalski, "Next decade in infrared detectors," Proc. SPIE 10433, 104330L (2017).

8. P. G. Lucey and B. B. Wilcox, "Mini-SMIFTS: an uncooled LWIR hyperspectral sensor," Proc. SPIE 5159, 275-282 (2003).

9. M. T. Eismann, Hyperspectral Remote Sensing, SPIE Press, Bellingham, Washington (2012).

10. P. K. Katti and K. Singh, "A note on the surface accuracy and alignment of the end mirrors in a Michelson interferometer," Appl. Opt. 5(12), 1962-1964 (1966).

11. W. He et al., "Polarization properties of a corner-cube retroreflector with three-dimensional polarization ray-tracing calculus," Appl. Opt. 52(19), 4527-4535 (2013).

12. D. G. Manolakis, R. B. Lockwood, and T. W. Cooley, Hyperspectral Imaging Remote Sensing, Cambridge University Press, University Printing House Cambridge (2016).

13. S. K. Dvoruk et al., "Effect of intrinsic radiation on the operation of a Fourier spectrometer," J. Opt. Technol. 70 (5), 317-321 (2003).

14. H. E. Revercomb et al., "Radiometric calibration of IR Fourier transform spectrometers: solution to a problem with the high-resolution interferometer sounder," Appl. Opt. 27(15), 3210-3218 (1988).

15. Y. Xiao et al., "Measuring thermal emission near room temperature using Fourier-transform infrared spectroscopy," Phys. Rev. Appl. 11, 014026 (2019). 
Renhorn, Svensson, and Boreman: Performance of an uncooled imaging interferometric spectrometer...

16. N. Matallah et al., "Design and first results of a Fourier transform imaging spectrometer in the 3-5 $\mu \mathrm{m}$ range," Proc. SPIE 8167, 816715 (2011).

17. R. Harig, "Passive remote sensing of pollutant clouds by Fourier-transform infrared spectrometry: signal-to-noise ratio as a function of spectral resolution," Appl. Opt. 43(23), 46034610 (2004).

Ingmar G. E. Renhorn is with Renhorn IR consultant AB, Sweden, and cofounder of Glana Sensors AB. He was a staff scientist at Swedish Defence Research Agency (FOI), Linköping, Sweden, from 1981 to 2014. In 1994, he was appointed as a director of research at the Department of IR Systems at FOI. He has been working as a consultant from 2014 to the present. His research interests include hyperspectral imaging systems, optical turbulence and aerosol profiling, signature measurement, and sensor systems with applications in reconnaissance, infrared search and track, target acquisition, and optical warning. He is a SPIE fellow and member of OSA.

Thomas Svensson is a senior scientist at the Swedish Defence Research Agency (FOI), Linköping, Sweden, where he has been working since 2001. His research interests include infrared sensors and topics such as hyperspectral imaging and night vision.

Glenn D. Boreman is a professor and chair of the Department of Physics and Optical Science and director of the Center for Optoelectronics and Optical Communications at the University of North Carolina at Charlotte. He is the cofounder and board chairman of Plasmonics, Inc., Orlando. From 1984 to 2011, he was on the faculty of the University of Central Florida, where he is now a professor Emeritus. He has supervised to completion $35 \mathrm{MS}$ and $27 \mathrm{PhD}$ students. $\mathrm{He}$ has held visiting research positions at IT\&T (Roanoke), Texas Instruments (Dallas), US Army Night Vision Lab (Ft. Belvoir), McDonnell Douglas Missile Systems (Titusville), US Army Redstone Arsenal (Huntsville), Imperial College (London), Universidad Complutense (Madrid), Swiss Federal Institute of Technology (Zürich), Swedish Defense Research Agency (Linköping), and University of New Mexico (Albuquerque). He received his BS degree in optics from the University of Rochester, and his $\mathrm{PhD}$ in optics from the University of Arizona. Boreman served as editor-in-chief of Applied Optics from 2000 to 2005 and deputy editor of Optics Express from 2014 to 2019. He is coauthor of the graduate textbooks Infrared Detectors and Systems and Infrared Antennas and Resonant Structures, and author of Modulation Transfer Function in Optical \& Electro-Optical Systems, 2nd ed. and Basic Electro-Optics for Electrical Engineers. He has published more than 200 refereed journal articles in the areas of infrared sensors and materials, optics of random media, and image-quality assessment. He is a fellow SPIE, the Institute of Electrical and Electronics Engineers (IEEE), the Optical Society (OSA), and the Military Sensing Symposium (MSS). He is a professional engineer registered in Florida. He served as the 2017 president of SPIE. 